\title{
A Synthetic, Xeno-Free Peptide Surface for Expansion and Directed Differentiation of Human Induced Pluripotent Stem Cells
}

\author{
Sha Jin ${ }^{1 *}$, Huantong Yao ${ }^{1}$, Jennifer L. Weber ${ }^{2}$, Zara K. Melkoumian ${ }^{2}$, Kaiming Ye ${ }^{1}$ \\ 1 Department of Biomedical Engineering, College of Engineering, University of Arkansas, Fayetteville, Arkansas, United States of America, 2 Corning Incorporated, Life \\ Sciences, Corning, New York, United States of America
}

\begin{abstract}
Human induced pluripotent stem cells have the potential to become an unlimited cell source for cell replacement therapy. The realization of this potential, however, depends on the availability of culture methods that are robust, scalable, and use chemically defined materials. Despite significant advances in hiPSC technologies, the expansion of hiPSCs relies upon the use of animal-derived extracellular matrix extracts, such as Matrigel, which raises safety concerns over the use of these products. In this work, we investigated the feasibility of expanding and differentiating hiPSCs on a chemically defined, xenofree synthetic peptide substrate, i.e. Corning Synthemax ${ }^{\circledR}$ Surface. We demonstrated that the Synthemax Surface supports the attachment, spreading, and proliferation of hiPSCs, as well as hiPSCs' lineage-specific differentiation. hiPSCs colonies grown on Synthemax Surfaces exhibit less spread and more compact morphology compared to cells grown on Matrigel ${ }^{\mathrm{TM}}$. The cytoskeleton characterization of hiPSCs grown on the Synthemax Surface revealed formation of denser actin filaments in the cell-cell interface. The down-regulation of vinculin and up-regulation of zyxin expression were also observed in hiPSCs grown on the Synthemax Surface. Further examination of cell-ECM interaction revealed that hiPSCs grown on the Synthemax Surface primarily utilize $\alpha_{v} \beta_{5}$ integrins to mediate attachment to the substrate, whereas multiple integrins are involved in cell attachment to Matrigel. Finally, hiPSCs can be maintained undifferentiated on the Synthemax Surface for more than ten passages. These studies provide a novel approach for expansion of hiPSCs using synthetic peptide engineered surface as a substrate to avoid a potential risk of contamination and lot-to-lot variability with animal derived materials.
\end{abstract}

Citation: Jin S, Yao H, Weber JL, Melkoumian ZK, Ye K (2012) A Synthetic, Xeno-Free Peptide Surface for Expansion and Directed Differentiation of Human Induced Pluripotent Stem Cells. PLoS ONE 7(11): e50880. doi:10.1371/journal.pone.0050880

Editor: Hani A. Awad, University of Rochester, United States of America

Received June 23, 2012; Accepted October 25, 2012; Published November 30, 2012

Copyright: ( 2012 Jin et al. This is an open-access article distributed under the terms of the Creative Commons Attribution License, which permits unrestricted use, distribution, and reproduction in any medium, provided the original author and source are credited.

Funding: This research was partially supported by National Science Foundation (NSF) grant CBET 0756455, Arkansas Biosciences Institute grant 0402-27504211108, 0402-27504-210012, and National Institutes of Health (NIH) funding 1P30RR031154 from the National Center for Research Resources (NCRR), a component of the National Institutes of Health. Its contents are solely the responsibility of the authors and do not necessarily represent the official view of NCRR or NIH. The funders had no role in study design, data collection and analysis, decision to publish, or preparation of the manuscript.

Competing Interests: Jennifer L. Weber and Zara K. Melkoumian are employed by Corning Incorporated Life Sciences. All other authors declare no further conflicts. This does not alter the authors' adherence to all the PLOS ONE policies on sharing data and materials.

*E-mail: sjin@uark.edu

\section{Introduction}

Unlike human embryonic stem cells (hESCs), human induced pluripotent stem cells (hiPSCs) can be derived from the patients' own cells. This raises hopes for generating patient-specific cells to treat many otherwise incurable diseases through cell replacement therapy $[1,2,3]$. Although the clinical application of hiPSCs is still at its early stage, the safety of these cell products has already received broad attention. For clinical use of these cells, it is highly desirable to have culture methods that are scalable and use chemically defined raw materials for both cell expansion and differentiation. The development of serum-free embryonic stem cell culture media, such as mTeSR, provides a chemically defined cell culture medium for expansion of hiPSCs [4,5]. However, development of chemically-defined culture surfaces to support hiPSCs growth and differentiation remains elusive.

Currently, hiPSCs are maintained and differentiated on either feeder layer cells [6] or Matrigel coated cell culture dishes $[7,8,9,10]$. Compared to feeder layer cells, the preparation of
Matrigel coated surfaces is a relatively easy and inexpensive process. However, Matrigel is an undefined mixture of extracellular matrix (ECM) proteins extracted from the Engelbreth-HolmSwarm (EHS) mouse sarcoma. It consists of laminin, collagen IV, heparan sulfate proteoglycans, entactin, nidogen, and some undefined factors. As a result, the quality and composition of Matrigel varies from lot to lot. There is also a risk of potential contamination with animal-derived viruses, raising significant safety concerns over Matrigel use in clinical applications [11]. Hence, there is a great need for the development of xeno-free, synthetic surfaces that capable of providing necessary stem cell niches to allow hiPSCs expansion and differentiation in a serumfree defined media.

It has been well documented that the in vitro expansion of stem cells relies on cell-ECM interaction, which occurs between cell surface adhesion molecules, such as integrins and their counterparts in ECM. This interaction enables cells to attach, spread, proliferate, migrate, and differentiate on a substrate. Accordingly, a chemically defined synthetic substrate can be developed by 
coating or functionalizing the substrate with chemically synthesized materials that mimic the ligands of cell surface adhesive molecules. Many examples of this approach are available in literatures. For example, the coating of a substrate with single or multiple ECM proteins has been explored for hESC maintenance $[12,13,14]$. While the coating of a substrate with one or two ECM proteins has been successful, it is not ideal because the production of recombinant ECM proteins is still very expensive. However, encouraged by these successes, the use of a motif rather than a full protein to support hiPSC growth and differentiation has been proposed and rigorously examined. A line of evidence reveals that the cell surface adhesive molecule binding motifs of ECM proteins play an imperative role in guiding cell attachment and spreading on a substrate coated with the ECM protein [15]. These motifs also transmit physiochemical signals to a cell thereby altering the cell's fate [16]. Thus, it is conceivable that surfaces displaying cell adhesion promoting motifs can support hiPSC attachment and growth by recapitulating integrin matrix engagement found in cellECM interaction. Most of these cell adhesion motifs are short peptides that can be chemically synthesized using xeno-free raw materials. For example, the use of small peptides to mimic the function of laminin, one of the critical ECM proteins, has been widely explored $[17,18,19]$. Laminin has at least 15 isoforms, each consisting of $\alpha, \beta$, and $\gamma$ chains [20]. The cell binding domains of laminin isoform 1 (laminin-1) has been characterized using fibroblasts adhesion model. As a result, four peptide sequences located on different laminin chains have been identified [16]. It has been shown by an integrin blocking assay that treatment of hES/iPS cells with anti-integrin $\alpha_{5} \beta_{1}, \alpha_{v} \beta_{3}$, or $\alpha_{v} \beta_{5}$ antibodies can suppress hESC attachment to the substrates coated with these peptides [15,21]. Despite the success in creating these synthetic substrates for hESC expansion and differentiation, the requirement of using either conditioned medium, which is not chemically defined [22,23,24], or an inhibitor of rho-associated kinase (ROCK), such as Y27632 [25,26], complicates the process. The use of ROCK inhibitor is not desirable for clinical applications. It has been shown that inhibiting ROCK could lead to kidney failure as well as acute hypotension caused by the relaxation of the vascular smooth muscles $[27,28]$. To overcome these issues, the Synthemax Surface has been recently developed by Corning Inc. It is based on the Arg-Gly-Asp (RGD) motif containing a short peptide, which is derived from the vitronectin protein and immobilized on the acrylate coating. The Synthemax Surface was shown to successfully support hESC growth and differentiation in chemically defined media $[11,29]$. This example proves that a short peptide can indeed recapitulate integrin ECM engagement found in hESCs grown on Matrigel-coated substrate.

The goal of this study was to determine whether hiPSCs can be maintained over a long period of time and differentiated on the Synthemax Surface. A line of evidence suggests that hiPSCs and hESCs exhibit some differences, despite similar patterns in global transcriptome assessment [30,31]. It has been found that a subset of 318 genes differentially expressed between these two types of cells [31]. This small set of genes may represent a genetic memory of the ancestor cells from which the hiPSCs were derived [30]. Thus, it is critical to assess whether the Synthemax Surface is suitable for hiPSC maintenance and differentiation. The underlying mechanisms of these processes have also been characterized in this study.

\section{Materials and Methods}

\section{1. hiPSC Expansion}

The hiPSC line IMR90 and Gibco ${ }^{\circledR}$ Episomal hiPSC Line were acquired from the WiCell Institute and Invitrogen, respectively. IMR90 cells were maintained on Matrigel (BD Biosciences, CA) coated polystyrene tissue culture treated dishes in a chemically defined medium mTeSR1 (Stem Cell Technologies, Vancouver, Canada) at $37^{\circ} \mathrm{C}$ and $5 \% \mathrm{CO}_{2}$ with daily medium exchange. The growth factor reduced Matrigel (BD Biosciences) was diluted 1:100 in a DMEM/F12 medium (HyClone, Utah) and coated to cell culture dishes for one hour. hiPSCs were seeded and cultured on Matrigel-coated dishes in the undifferentiated state. Similarly, Gibco hiPSC were maintained on Geltrex ${ }^{\mathrm{TM}}$ (Gibco) coated polystyrene tissue culture treated dishes in mTeSRl prior to expansion on Synthemax ${ }^{\circledR}$ surface (Corning, NY). Subculture of hiPSGs was performed by treating these cells with $1 \mathrm{mg} / \mathrm{mL}$ dispase (Stem Cell Technologies), followed by a gentle scraping of cell colonies from the culture dishes with a split ratio of $1: 3$ to 1:5 every three or four days. hiPSCs were also seeded onto a synthetic peptide functionalized Synthemax Surface (Corning, NY) at a density of 50,000 cells $/ \mathrm{cm}^{2}$ in the mTeSR 1 medium. To determine hiPSC expansion on these surfaces, cells were harvested with $0.05 \%$ trypsin-EDTA for $5 \mathrm{~min}$, and the total cell number, as well as the viable cell number, was counted using a Cell Counter (Bio-Rad) following trypan blue staining. Cell morphology was monitored daily to insure that the cells maintained an undifferentiated state. To monitor genomic integrity, cell samples for all conditions were submitted for karyotype analysis by G-banding (WiCell Research Institute Cytogenetics Lab).

\subsection{Induction of hiPSC Differentiation}

To induce definitive endoderm (DE) differentiation, undifferentiated hiPSCs were seeded onto Matrigel and Synthemax Surfaces and cultured overnight in the mTeSR medium after dispase treatment $(1 \mathrm{mg} / \mathrm{mL})$ and gentle scraping. Cells were then fed with a definitive endoderm (DE) induction medium containing RPMI1640, B27 (Invitrogen), $1 \mathrm{mM}$ sodium-butyrate, and $4 \mathrm{nM}$ activin $\mathrm{A}$. The sodium butyrate concentration was reduced to $0.5 \mathrm{mM}$ after $24 \mathrm{~h}$ differentiation. The differentiation medium was changed every other day until day 7 post differentiation induction. To induce embryoid body (EB) formation, hiPSC colonies were harvested after thirteen passages on Synthemax Surface, and seeded onto ultra-low attachment six-well plates for EB formation assay. Cells were maintained in Iscove's Modified Dulbecco's Medium (IMDM) supplemented with 10\% FBS and re-fed every 2 days for 14 days. EBs were frozen in CryoStor-10 cryopreservation medium. qRT-PCR was performed on EBs to confirm the presence of the three germ layer markers.

\subsection{Immunofluorescence Microscopy}

To examine the protein expression in hiPSCs, immunofluorescent staining was performed as described in our previous work [32]. Mouse anti-human octamer-binding transcription factor 4 (OCT4) (1:100), Stage-specific embryonic antigen-4 (SSEA4) (1:100) (Millipore, Billerica, MA), SOX17 (1:50; R\&D Systems); rabbit anti-human FOXA2 (1:1000; Abcam), vinculin (1:50; Santa Cruz, CA), zyxin (1:100; Sigma), and Alexa Fluor 488 phalloidin ( $5 \mathrm{U} / \mathrm{ml}$, Invitrogen) were used as primary antibodies. The goat anti-mouse Alexa Fluor $488 \mathrm{IgG}_{1}$ (1:200), goat anti-mouse Alexa Fluor $488 \mathrm{IgG}_{3}$ (1:200) (Life Technologies, Carlsbad, CA), goat anti-mouse IgG FITC (1:100; Sigma), donkey anti-rabbit IgG TRITC (1:50) (Jackson Immuno research Laboratories In.), and mouse anti-rabbit IgG FITC antibodies (1:150; Sigma) were used 


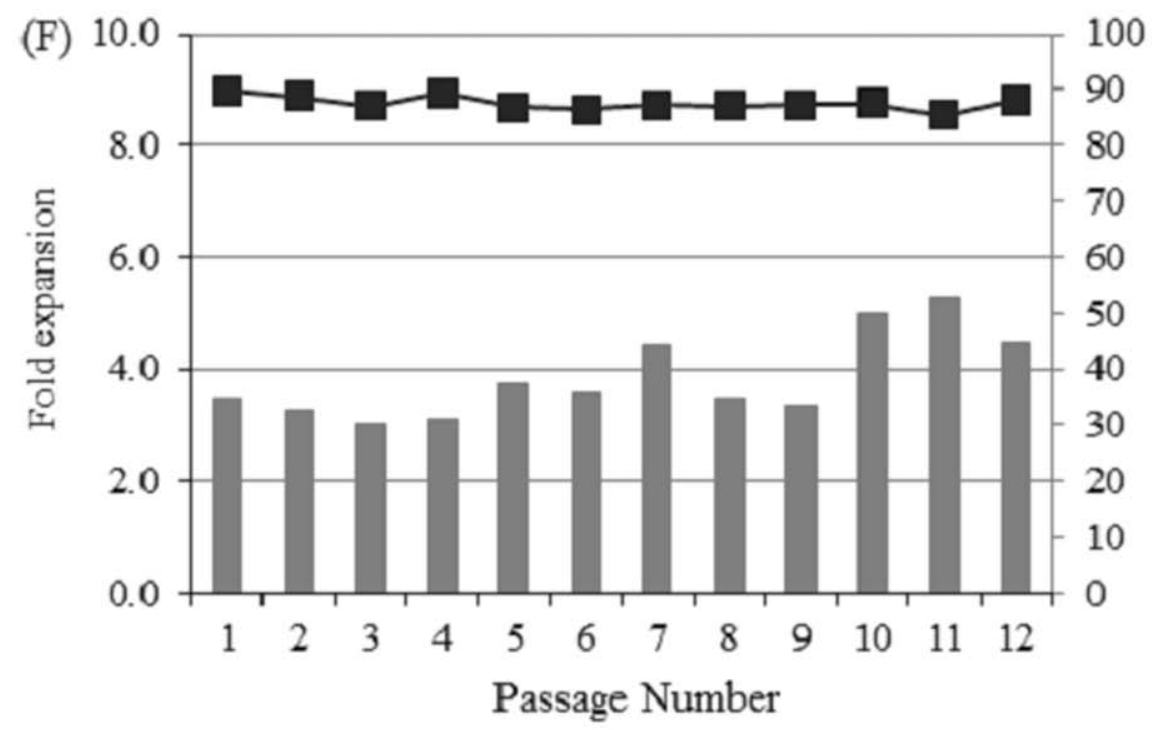

疍

(G)
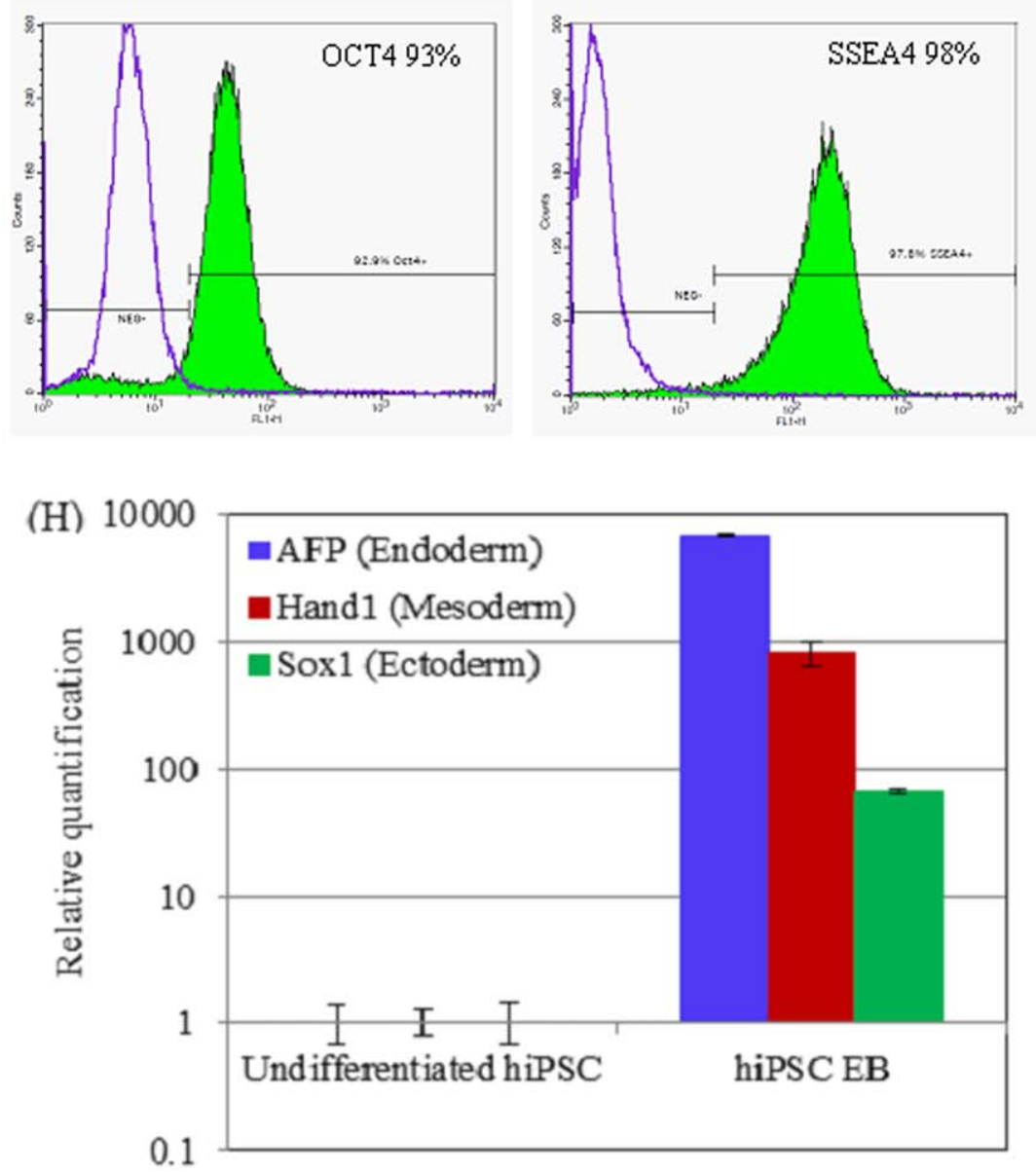

Figure 1. Comparison of hiPSC proliferation on Synthemax Surface and Matrigel. Brightfiled image of hiPSC colony formed on SM (A) and MG (B) surface. Scale bar: $50 \mu \mathrm{m}$. (C) Cell proliferation on different surfaces determined by counting viable cell number every $24 \mathrm{~h}$. Data is presented as mean \pm SD from three independent experiments. p-value: 0.7008 (D) Detection of pluripotency of hiPSCs on Synthemax Surface by quantitative real-time PCR analysis. The expression level of pluripotent marker gene in cells maintained on Matrigel-coated surface was detected for comparison. (E) Immunofluorescent staining of stem cell pluripotency markers, OCT4 and SSEA4 in hiPSCs maintained on SM surface for ten passages. Scale bar: $100 \mu \mathrm{m}$. MG, Matrigel; SM, Synthemax Surface. (F) Cell expansion and viability in the course of twelve consecutive passages on Synthemax Surface. (G) Flow cytometry analysis of OCT4 and SSEA4 markers after ten passages of hiPSCs on Synthemax Surface. (H) qPCR analysis of germ layer markers after induced differentiation of hiPSC maintained on Synthemax Surface for more than ten passages. doi:10.1371/journal.pone.0050880.g001 


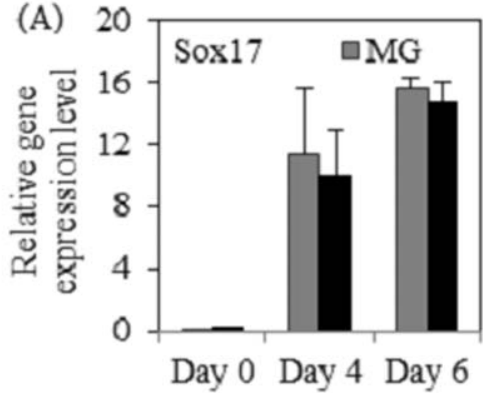

(C) DAPI
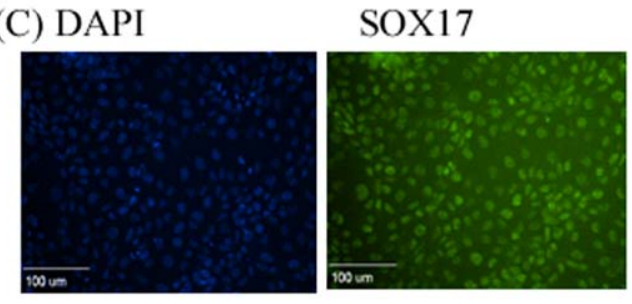

(D) $200 \mathrm{X}$

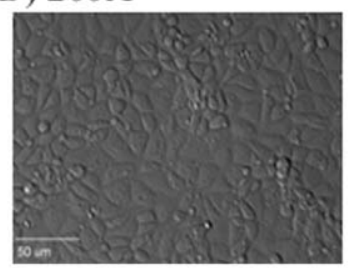

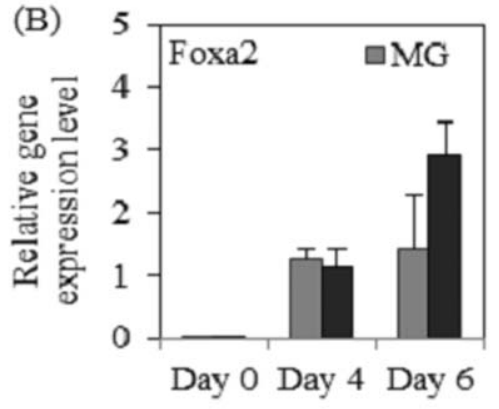

FOXA2

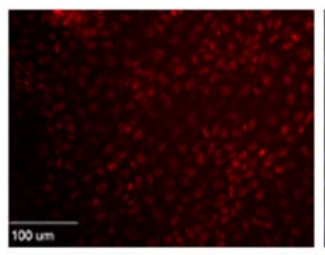

DAPI/SOX17/FOXA2

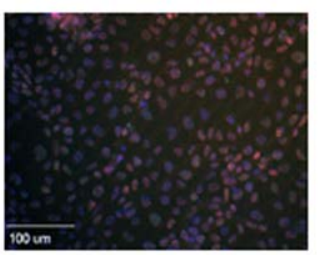

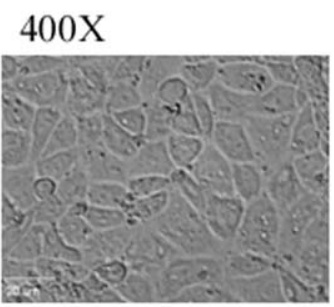

Figure 2. Definitive endoderm (DE) differentiation of hiPSC on Matrigel and Synthemax Surface. The expression of SOX17 (A) and FOXA2 (B) was normalized to their expression levels in adult pancreatic (AP) tissues. Data is presented as mean \pm SD from three independent experiments. $p$-value $=0.917$ for SOX17 and $p$-value $=0.668$ for FOXA2. (C) Immunofluoresent staining for SOX17 and FOXA2 DE markers in hiPSCs differentiated on SM. (D) Phase contrast images of hiPSC morphology after DE differentiation on SM. Majority of the cells exhibited endothelial-like morphology on day 6 post differentiation.

doi:10.1371/journal.pone.0050880.g002

as the secondary antibodies. Cells were labeled with DAPI (4',6diamidino-2-phenylindole) nuclear stain. Four drops of DAPI (Vector Laboratories, Inc. Burlingame, GA) were added to the each well and incubated for one minute. The specificities of these antibodies were verified against their corresponding isotype controls. With the Slidebook imaging software, we first optimized the exposure time and fluorescence sensitivity by using our negative control samples, to ensure no auto-fluorescence or false positive imaging. The same parameters were then used for imaging the rest of the samples.

\subsection{Adhesion-blocking Assay}

Adhesion-blocking assays were carried out as described previously with some modifications [13]. In brief, approximately $0.5 \times 10^{6}$ hiPSCs were incubated in a phenol red-free CMRL 1066 medium (Cellgro) containing $0.35 \%$ bovine serum albumin (BSA) at $37^{\circ} \mathrm{C}$ for $30 \mathrm{~min}$ in the absence or presence of mouse antihuman integrin antibodies $(10 \mu \mathrm{g} / \mathrm{ml}$, Chemicon International, Billerica, MA). After incubation, cells were seeded onto either Synthemax Surface (SM) or Matrigel (MG) coated 6-well plates. The cells were allowed to adhere to the surfaces at $37^{\circ} \mathrm{C}$ in a $5 \%$ $\mathrm{CO}_{2}$ incubator for $1 \mathrm{~h}$. Nonattached cells were carefully removed by washing the wells three times with CMRL/ $0.35 \%$ BSA and once with PBS. The attached cells were fixed with $100 \%$ ethanol at room temperature for $5 \mathrm{~min}$ and stained with $0.4 \%$ crystal violet in methanol for $5 \mathrm{~min}$, followed by a thorough wash with MilliQ water. Cell attachment was visually scored by counting the number of cell colonies in seven randomly selected fields. The average of these numbers was normalized to a non-blocking control and used as an index for quantifying cell attachment. The microscopy images were captured using an inverted Olympus IX71 microscope (Tokyo, Japan) equipped with a cooled chargecoupled device (CCD) camera (Qimaging Retiga 4200, Surrey, Canada) controlled by a Lambda 10-3 (Sutter Instrument Company, CA).

\subsection{Quantitative Real-time PCR (qRT-PCR)}

TaqMan quantitative real-time (qRT-PCR) analysis was performed according to our previous work $[32,33]$. Briefly, total RNA was extracted from cells using an RNeasy kit (Qiagen, Valencia, CA). The genomic DNAs were eliminated from RNA samples during extraction. After quantifying with a Synergy MX microplate reader (BioTek, Winooski, VT), qRT-PCR was performed to detect pluripotent stem cell (OCT4) and differentiation marker genes with TaqMan Master Mix from Applied Biosystems, as described elsewhere [32]. The following primer-probe pairs were used for mRNA detection. OCT4: Forward 5'- GAAACCCACACTGCAGCA $\quad-3^{\prime}$, Reverse 5'-CACATCGTTCTCGAGCCCA-3', and probe 5' FAM -CAGCCACATCGCCCAGCA - BHQ1 3'. The following TaqMan ${ }^{\circledR}$ Assay IDs were used to detect all three germ layer markers: AFP: Hs00173490_ml; Handl: Hs02330376_sl; SOX1: Hs01057642_sl (Invitrogen). The RNA samples that were not reversely transcribed were used as controls in order to rule out any 
(A)

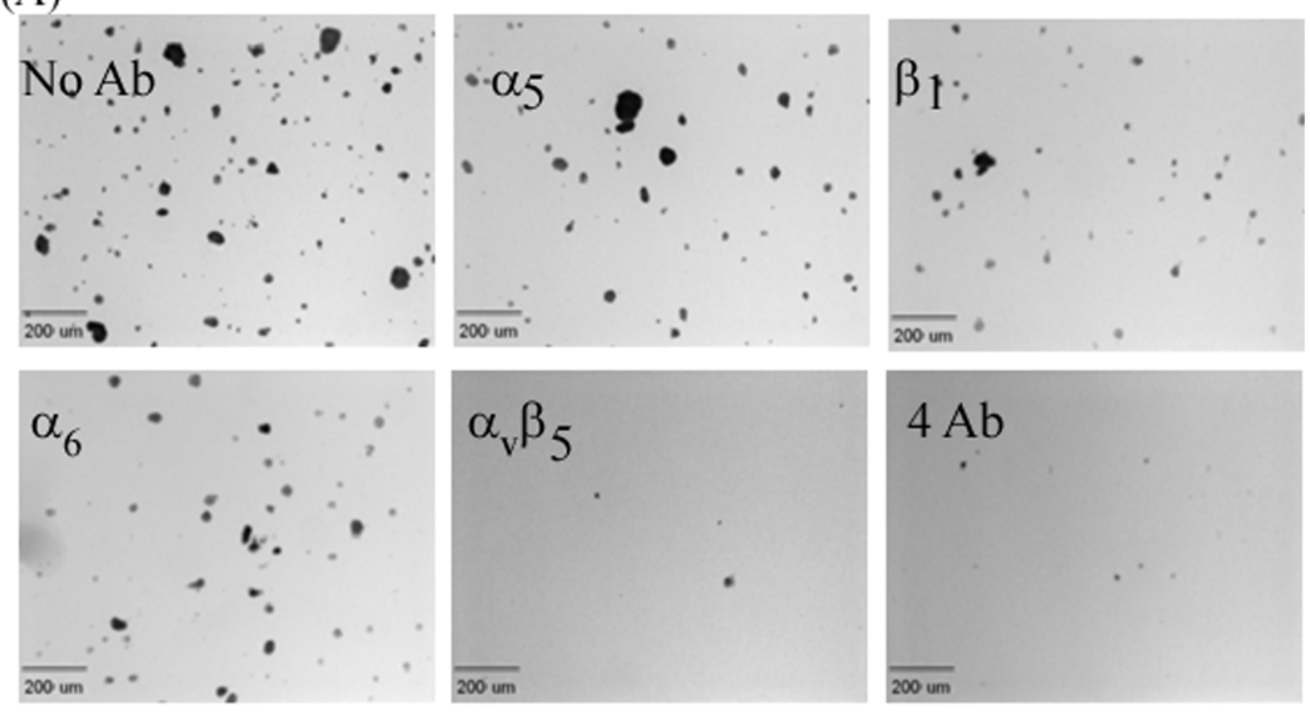

(B)

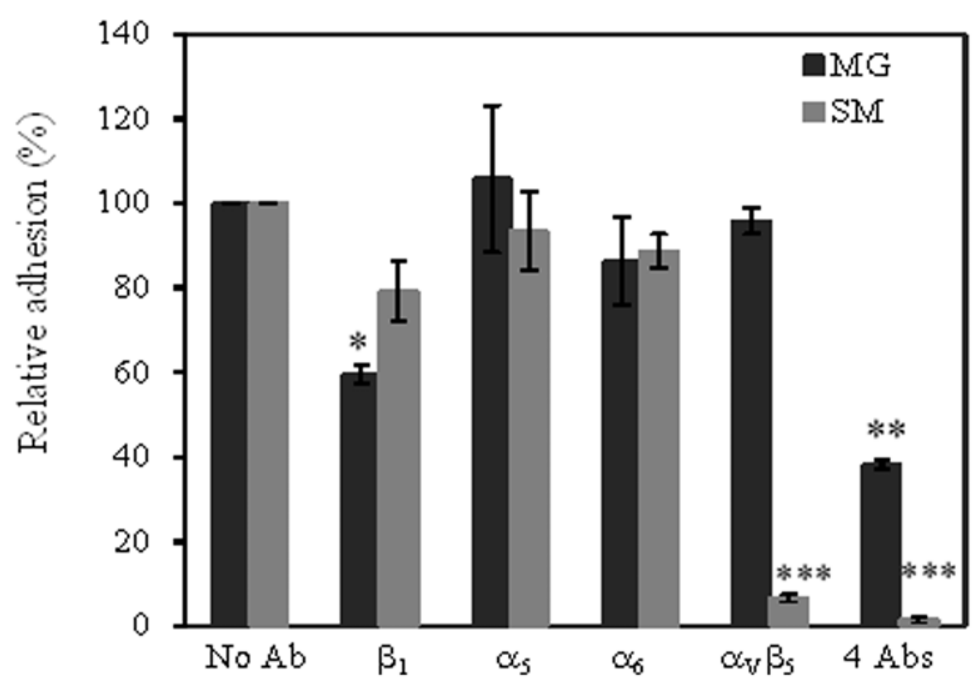

Figure 3. The role of integrin receptors in hiPSC adhesion to Synthemax Surface and Matrigel. hiPSCs were incubated with indicated anti-integrin antibodies before seeding onto the corresponding surface. (A) Micrographic images of cell attachment to the Synthemax Surface after integrin treatment. Scale bar: $200 \mu \mathrm{m}$. (B) Relative cell adhesion determined at $1 \mathrm{~h}$ post seeding. A cell adhesion was estimated by counting the number of colonies in 14 randomly selected fields. The relative cell adhesion was normalized to that for integrin-untreated cells. All results were expressed as the mean $\pm S D(n=14)$ in two typical measurements of more than 3 independent experiments. ${ }^{*}: p=0.0093 ;{ }^{* *}: p=0.0002 ;{ }^{* *}$ : $\mathrm{p}<2.75 \mathrm{E}-10$. Symbols: Ab, antibody; MG, Matrigel; SM, Synthemax Surface.

doi:10.1371/journal.pone.0050880.g003

possibilities of genomic DNA contamination in the qRT-PCR assay.

\subsection{Western Blot}

To characterize cytoskeletal protein expression, total cell proteins were extracted using a cell lysis buffer containing $50 \mathrm{mM}$ Tris-HCl (pH 7.4), $1 \%$ Triton X-100, 0.1\% SDS, $150 \mathrm{mM} \mathrm{NaCl}, 1 \mathrm{mM}$ Dithiothreitol, and $1 \mathrm{mM}$ phenylmethylsulfonyl fluoride. Western blot was carried out as described in our previous work [34,35]. Rabbit anti-human $\boldsymbol{\alpha}$-actinin (1:1000; Sigma), anti-human zyxin (1:1000; Sigma), and anti-vinculin antibodies (1:200; Santa Cruz) were used as primary antibodies, whereas goat anti-rabbit IgG horseradish peroxidase (HRP) conjugates were used (1:1000; Sigma) as secondary antibodies. $\beta$-actin served as a control for semi-quantitative analysis of
Western blot. It was detected using mouse HRP-conjugated $\beta$ actin antibodies (1:35,000; Sigma).

\subsection{Statistical Analyses}

Data were presented as mean \pm standard deviation. The statistical analysis was performed based on ANOVA and post-hoc significant difference test. Statistical significance was determined at p0.05.

\section{Results}

3.1. Attachment, Spreading, and Proliferation of hiPSCs on Chemically Defined Synthetic Peptide Surface

First, we examined whether hiPSCs can attach, spread, and proliferate on the Synthemax Surface. hiPSCs were seeded onto 

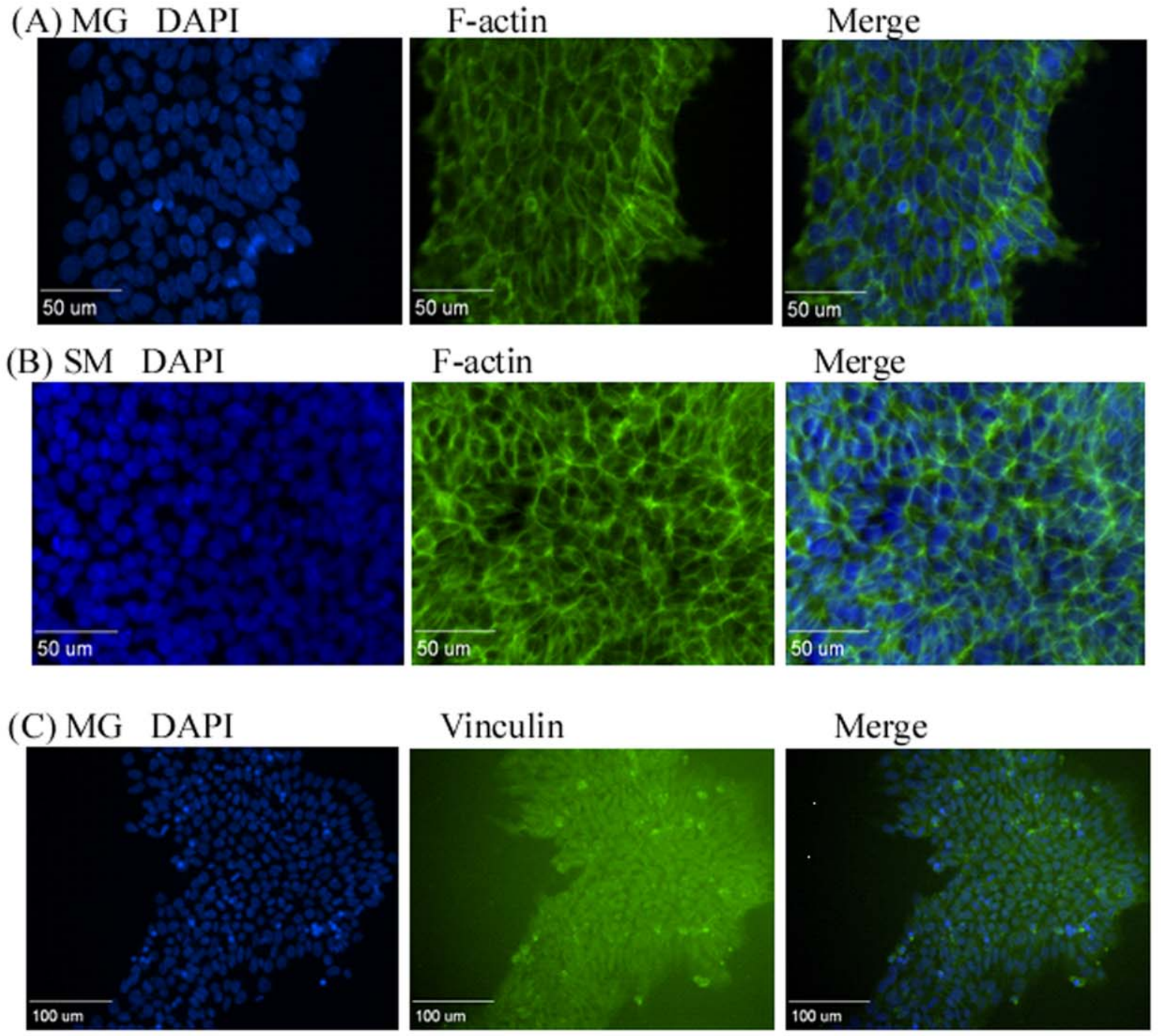

(D) SM DAPI

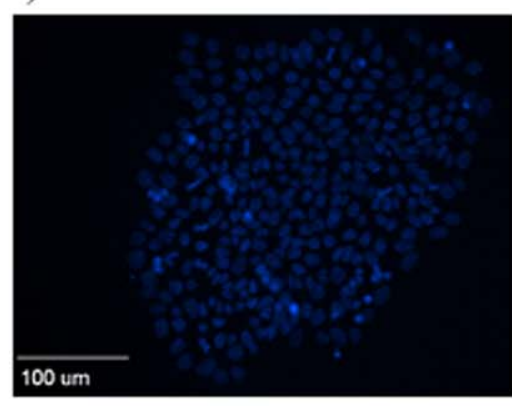

Vinculin

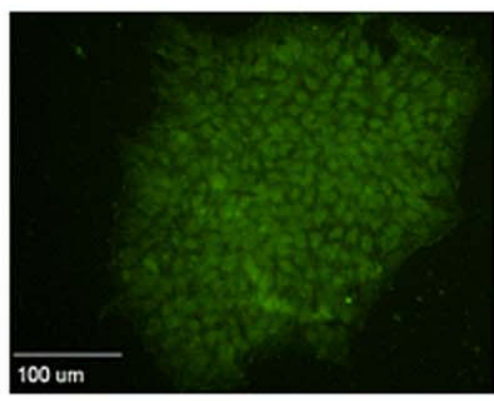

\section{Merge}

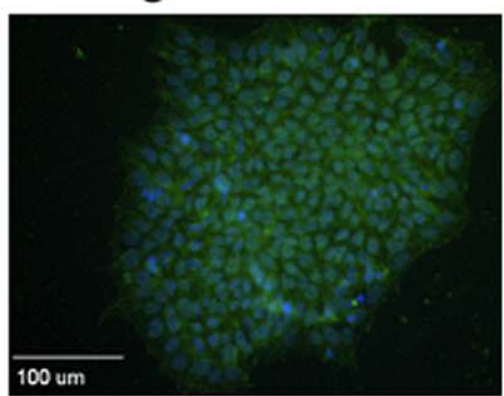

Figure 4. Organization of cytoskeletal structure proteins in hiPSCs grown on different surfaces. (A, C, E): MG surface; (B, D, F): SM surface. hiPSCs were fixed after growing on the corresponding surfaces for two days and stained with antibodies against F-actin, vinculin, and zyxin, respectively. Arrows indicate denser and more pronounced actin filament expression. Scale bars: (A-B, E-F) $50 \mu \mathrm{m},(C-D) 100 \mu \mathrm{m}$. MG, Matrigel; SM, Synthemax Surface.

doi:10.1371/journal.pone.0050880.g004

both Synthemax and Matrigel surfaces and cultured in a chemically defined mTeSR 1 medium. The attachment of hiPSGs to the surfaces was observed and imaged using an inverted phase contrast microscope, whereas cell proliferation was determined by counting viable cells every 24 hours until day 4 . The observation of cell colony morphology on these surfaces revealed that cell colonies on the Synthemax Surface became more compact than those on Matrigel (Figs. 1A and B). This was supported by counting the number of viable cells per $\mathrm{cm}^{2}$ for both surfaces. As shown in Fig. 1C, a higher cell number was observed on Synthemax Surface. The cell doubling time on Synthemax Surface was 41.2 hours, compared to 43.6 hours on Matrigel. ANOVA statistical test shows p-value of 0.7008 , indicating there is 
(A)

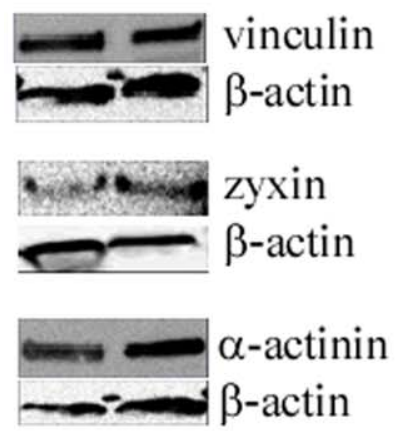

(B)

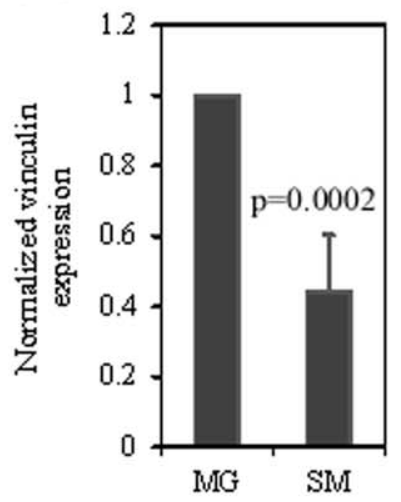

(C)

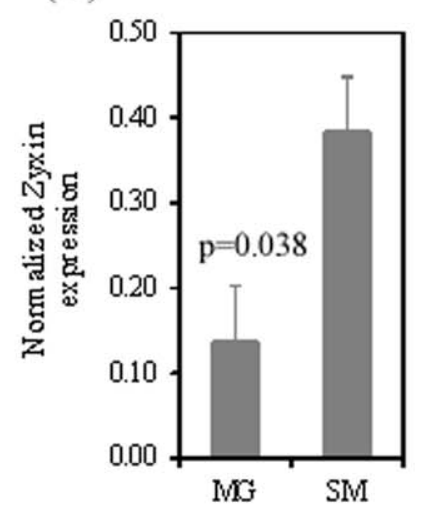

(D)

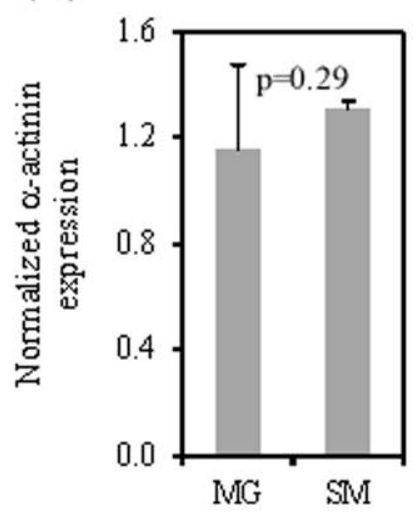

Figure 5. Cytoskeletal protein expression in hiPSCs grown on Matrigel and Synthemax surface. Cells were harvested at $48 \mathrm{~h}$ post seeding and total proteins were extracted for Western blot analysis. At least three independent experiments were performed and data were presented as mean \pm SD. (A) Vinculin, zyxin, and $\alpha$-actinin expression detected by Western blot analysis. (B-D) Relative protein expression using $\beta$ actin as a loading control. Semi-quantification of protein expression was performed by Kodak 1D gel imaging software. MG, Matrigel; SM, Synthemax Surface.

doi:10.1371/journal.pone.0050880.g005

no significant difference between doubling time for cells cultured on Matrigel and Synthemax Surface.

Next, we determined whether hiPSCs can be maintained in the undifferentiated state on the Synthemax Surface over the course of multiple passages. OCT4 mRNA expression in cells cultured on Synthemax Surface after one passage and ten passages were analyzed and compared with the expression level in cells cultured on Matrigel-coated surface (Fig. 1D). The OCT4 mRNA expression was similar for cells on both surfaces. We did not observe significant difference among the samples tested ( $p$ value $=0.5190)$. In addition, immunofluorescent microscopy was performed to monitor the expression of embryonic stem cell pluripotency markers: OCT4 and stage-specific embryonic antigen 4 (SSEA4) in hiPSCs maintained on Synthemax Surface. As presented in Fig. 1E, hiPSCs sustained a high level of expression of OCT4 and SSEA4 after ten passages, suggesting their pluripotent status. The same results were demonstrated with the second hiPSC line. As show in Figure 1F, hiPSC were successfully maintained on Synthemax Surface in mTeSRl medium for more than ten passages with the stable fold expansion and high viability. After ten passages on Synthemax Surface, hiPSC maintained high level of pluripotency markers, OCT4 and SSEA4 (Figure 1G) and ability to differentiate into all three germ layers (Figure $1 \mathrm{H}$ ). Together, these data demonstrate that hiPSCs can self-renew on Synthemax Surface for more than ten passages without losing their immunophenotype and pluripotency.

\subsection{Directed Differentiation of hiPSC on Synthetic Peptide Surface}

We next investigated whether hiPSCs retain their ability to differentiate into a specific lineage, such as a DE lineage. The differentiation of DE lineage is the first and also the most critical step in hESC pancreatic differentiation [36,37,38]. Thus, demonstration of hiPSC directed DE differentiation on peptide surface could help develop a xeno-free differentiation system to generate transplantable $\beta$-cells from hiPSCs for diabetes therapy. To perform DE differentiation, the hiPSCs were cultured on the Synthemax Surface followed by induction of DE differentiation using special medium described in Materials and Methods section. The expressions of DE markers SOX17 and FOXA2 in differentiated hiPSCs were detected by qRT-PCR. As presented in Fig. 2, similar expression levels of the marker genes were observed in cells differentiated on either Synthemax or Matrigel surfaces since statistical test indicated p-value of 0.917 and 0.668 , respectively, for SOX17 and FOXA2. The qRT-PCR results are supported by immunofluorescent microscopy analysis (Fig. 2E). The expressions of DE marker proteins for SOX17 and FOXA2 were observed in hiPSCs differentiated on the Synthemax Surface (Fig. 2C). The DE morphology was observed on day 5 post induction of differentiation (Fig. 2D). Our results indicate that the Synthemax Surface provides the appropriate niche environment that supports both the expansion and the directed differentiation of hiPSCis.

\subsection{Integrin $\alpha_{v} \beta_{5}$ Plays an Essential Role in hiPSCs Adhesion to the Synthetic Peptide Surface}

The success in expanding and differentiating hiPSCs on the synthetic peptide surface posed a fundamental question about the molecular mechanisms of cell attachment to this surface. We hypothesized that hiPSCs attach to the synthetic peptide substrate through integrin engagement. To identify which integrins are involved in mediating hiPSC attachment to the Synthemax Surface, we performed the adhesion inhibition assay by blocking various integrins with anti-integrin antibodies before seeding the cells to the Synthemax Surface. Cells seeded on the Matrigel substrates served as control for this assay. We found that the blocking of integrin $\alpha_{V} \beta_{5}$ significantly inhibited the attachment of hiPSCis to the Synthemax Surface by $93 \%$ (Fig. 3A). On the other hand, no significant reduction in cell adhesion by $\alpha_{V} \beta_{5}$ antibody was observed for hiPSCs on Matrigel surface (Fig. 3B). The blocking of integrins $\alpha_{5}, \alpha_{6}$, and $\beta_{1}$ only reduced the cell adhesion to the Synthemax Surface by 20,6 , and $11 \%$, respectively. Cells treated with all four antibodies against integrins $\alpha_{5}, \alpha_{6}, \beta_{1}$, and $\alpha_{V} \beta_{5}$ completely abolished the attachment of hiPSCs to the Synthemax Surface. These results suggest that hiPSCs interact with the Synthemax Surface mainly through the integrin $\alpha_{V} \beta_{5}$. Our results are consistent with previous studies that demonstrated recognition of recombinant vitronectin protein by integrin $\alpha_{\mathrm{V}} \beta_{5}$ $[13,39]$ since the Synthemax Surface contains peptide sequence derived from vitronectin proteins.

In contrast, the blocking of integrin $\beta_{1}$ led to $40 \%$ reduction in hiPSC adhesion to the Matrigel surface, indicating that integrin $\beta_{1}$ 
plays an important role in hiPSC attachment to Matrigel. The results are similar to the observation reported previously, which found that integrin $\beta_{1}$ is required for hiPSCs adhesion and proliferation on Matrigel-coated surfaces [39]. The blocking of integrin $\alpha_{6}$ resulted in a $14 \%$ reduction in hiPSC adhesion to Matrigel. The combination of antibodies against integrins $\alpha_{5}, \alpha_{6}$, $\beta_{1}$, and $\alpha_{V} \beta_{5}$ resulted in a $62 \%$ reduction of cell adhesion to the Matrigel surface. These results suggest that multiple integrins are involved in mediating hiPSCs adhesion to the Matrigel surface.

\subsection{Organization of Cytoskeleton Structures of hiPSCs Grown on the Synthemax Surface}

To investigate physicochemical cues provided by the Synthemax Surface for hiPSC proliferation and differentiation, we examined the organization of the cytoskeleton structures, such as actin filaments (F-actin) and vinculin during hiPSC growth on this surface. Actin filaments are the smallest filamentous proteins involved in both cell structure (a static role) and cell movement (a dynamic role). They are the main components of the cytoskeleton. Vinculin is another cytoskeletal protein that is recruited from the cytoplasm to the focal adhesions. Its recruitment is regulated by either external, internal, or both signals [40]. This recruitment is critical for cell spreading and migration [41,42]. To investigate actin polymerization in hiPSCs grown on the Synthemax Surface, cells were fixed, permeabilized, and labeled with phalloidin after 48 hours in culture. As shown in Fig. 4, hiPSGs grown on the Synthemax Surface exhibited the actin filament network that was significantly different from that formed in cells grown on the Matrigel surface. While cells grown on both surfaces showed actin stress fibers at focal adhesions, the accumulation of denser and broader actin filaments between the cell-cell interfaces occurred only in cells grown on the Synthemax Surface. This may suggest the enhanced actin filament networks in hiPSCs when they are grown on the Synthemax Surface. Vinculin staining revealed its comparable distribution in hiPSCs grown on both Synthemax and Matrigel surfaces (Fig. 4C \& D). However, cells grown on the Synthemax Surface expressed less vinculin, as revealed by a Western blot analysis (Fig. 5).

In addition, we found that there is a higher expression of zyxin protein in these cells. Zyxin is a zinc-binding phosphoprotein that concentrates at focal adhesions and along the actin cytoskeleton. The immunofluorescent microscopy showed that hiPSCs expressed and formed polygonal structures on both Synthemax and Matrigel surfaces (Fig. 4E). However, a semi-quantitative Western blot assay revealed a significant up-regulation of zyxin in cells grown on the Synthemax Surface (Fig. 5). Because zyxin is directly involved in cell spreading and proliferation and is inversely correlated to differentiation [43], its up-regulation may contribute to enhanced cell attachment and proliferation on the Synthemax Surface, as described above. Finally, $\alpha$-actinin expressions in cells grown on the Synthemax and Matrigel surfaces were comparable (Fig. 5D).

\section{Discussion}

In vivo, stem cells interact with various microenvironments, including tissue niches that regulate gene expressions, thus affecting cell fate. These niches can be categorized into three groups. The first group is signaling molecules, such as transforming factor- $\beta$ (TGF- $\beta$ ) superfamily members and fibroblast growth factors (FGFs). These signaling molecules regulate stem cell selfrenewal by activating Smad2/3 signaling and suppressing BMP (bone morphogenetic protein) signaling $[44,45,46,47,48,49]$. The second group is extracellular structures that support cell attach- ment, interaction, and migration. These local cellular microenvironments act on stem cells in an indirect fashion mediated by cellECM and/or cell-cell contacts [50]. The third group is physicochemical conditions to which stem cells are exposed. These physicochemical factors include $\mathrm{pH}$, ionic strength, oxygen level of a cell culture medium, and the mechanical/chemical properties and topographic features of a substrate that supports cell growth. Cells also generate their own mechanical signals through cell traction actions. These actions are driven by forces generated from different molecular interactions that are primarily mediated by the motor protein myosin II and other cytoskeletal systems, such as microtubules [51,52,53,54,55].

Therefore, for optimal expansion and differentiation of stem cells for clinical applications, it is critical to design a cell culture environment that mimics cell niches using defined xeno-free materials. In this work, we showed that the synthetic, xeno-free peptide surface, Synthemax Surface, supports long-term maintenance and directed differentiation of hiPSCs in a defined medium. This will offer a better solution to produce clinical-grade hiPSCs and their derivatives for therapeutic applications. We observed that hiPSC colonies formed on a peptide surface exhibit more compact morphology than those on Matrigel surface. The characterization of cell-ECM interaction in hiPSCs grown on the Synthemax Surface revealed that the integrin $\alpha_{V} \beta_{5}$ plays a key role in mediating the interaction between hiPSCs and the Synthemax Surface, which is functionalized with synthetic RGD-containing peptide sequence from vitronectin protein. In contrast, we found that cells grown on Matrigel coated surface utilize multiple integrins for mediating cell-ECM interaction. These results are consistent with other reports in the literature [15]. Vuoristo et al showed that blocking integrin $\beta_{1}$ subunit can completely obstruct the adhesion of hESCs to a basement membrane protein coated substrate [56]. Meng and co-workers found that the integrin $\alpha_{6}, \beta_{1}, \alpha_{2} \beta_{1}$ and $\alpha_{v} \beta_{3}$ regulate hESC attachment to Matrigel coated substrates in a chemically defined medium [15]. In summary, our results demonstrated that the engagement of a single $\alpha_{v} \beta_{5}$ integrin on the cell surface was sufficient to maintain the self-renewal of hiPSCs on the chemically-defined animal-free Synthemax Surface.

Cytoskeleton is a mechanotransduction component that is related to integrin signaling transduction pathways. The cytoplasmic domains of integrins bind to the cytoskeleton through adapter proteins like vinculin, $\alpha$-actinin, and phosphorylated-focal adhesion kinase (p-FAK). These protein complexes form focal adhesions, i.e., sub-cellular sites that receive and transduce physiochemical signals from a substrate $[57,58]$ to influence cell morphology [59,60], adhesion [61], proliferation [62], motility [63], differentiation [64], and cell fate [55]. The cytoplasmic domains of $\beta$-integrin also interact with talin, filamin, tesin, and other focal adhesion proteins to stabilize or destabilize focal adhesions $[55,65,66,67]$, leading to the remodeling of microfilament and microtubule networks, which in turn affects gene transfer and expression. We observed similar level of $\alpha$-actinin expression on both the Synthemax and the Matrigel surfaces, but found down-regulation of vinculin and up-regulation of zyxin in hiPSCs grown on the Synthemax Surface (Fig. 5). While the mechanism of these changes in cell cytoskeletal proteins is unclear, it may still indicate a reorganization of cellular molecules and focal adhesions, which facilitates the spreading and self-renewal of hiPSCs on substrates, such as peptide surface used in this work.

Another important finding of this study was that a synthetic Synthemax Surface not only supports hiPSC attachment, spreading, and differentiation; but also allows for long-term hiPSC selfrenewal in a defined medium. We demonstrated that hiPSCs 
retained stable proliferation and pluripotency markers after growth on the Synthemax Surface for ten consecutive passages. Our experimental results suggest that Synthemax Surface in combination with defined medium can provide a defined culture system for expansion of hiPSC for cell therapy applications.

\section{Acknowledgments}

We thank Dr. Yulong Hong and Paula Dolley-Sonneville from Corning Incorporated for their help with qPCR and flow cytometry analysis, respectively.

\section{References}

1. Thomson JA, Itskovitz-Eldor J, Shapiro SS, Waknitz MA, Swiergiel JJ, et al. (1998) Embryonic stem cell lines derived from human blastocysts. Science 282: 1145-1147.

2. Yu J, Hu K, Smuga-Otto K, Tian S, Stewart R, et al. (2009) Human induced pluripotent stem cells free of vector and transgene sequences. Science 324: 797801.

3. Takahashi K, Yamanaka S (2006) Induction of pluripotent stem cells from mouse embryonic and adult fibroblast cultures by defined factors. Cell 126: 663676.

4. Hannoun Z, Fletcher J, Greenhough S, Medine C, Samuel K, et al. (2010) The comparison between conditioned media and serum-free media in human embryonic stem cell culture and differentiation. Cell Reprogram 12: 133-140.

5. Ludwig T, J AT (2007) Defined, feeder-independent medium for human embryonic stem cell culture. Curr Protoc Stem Cell Biol Chapter 1: Unit 1C 2.

6. Ye K, Jin S, editors (2011) Human Embryonic and Induced Pluripotent Stem Cells Lineage-Specific Differentiation Protocols. 1st ed. New York: Humana Press.

7. Takahashi K, Tanabe K, Ohnuki M, Narita M, Ichisaka T, et al. (2007) Induction of pluripotent stem cells from adult human fibroblasts by defined factors. Cell 131: 861-872.

8. Ludwig TE, Levenstein ME, Jones JM, Berggren WT, Mitchen ER, et al. (2006) Derivation of human embryonic stem cells in defined conditions. Nat Biotechnol 24: 185-187.

9. Amit M, Shariki C, Margulets V, Itskovitz-Eldor J (2004) Feeder layer- and serum-free culture of human embryonic stem cells. Biol Reprod 70: 837-845.

10. Stewart MH, Bendall SC, Bhatia M (2008) Deconstructing human embryonic stem cell cultures: niche regulation of self-renewal and pluripotency. J Mol Med (Berl) 86: 875-886.

11. Melkoumian Z, Weber JL, Weber DM, Fadeev AG, Zhou Y, et al. (2010) Synthetic peptide-acrylate surfaces for long-term self-renewal and cardiomyocyte differentiation of human embryonic stem cells. Nat Biotechnol 28: 606-610.

12. Fu X, Toh WS, Liu H, Lu K, Li M, et al. (2011) Establishment of clinically compliant human embryonic stem cells in an autologous feeder-free system. Tissue Eng Part C Methods 17: 927-937.

13. Braam SR, Zeinstra L, Litjens S, Ward-van Oostwaard D, van den Brink S, et al. (2008) Recombinant vitronectin is a functionally defined substrate that supports human embryonic stem cell self-renewal via alphavbeta5 integrin. Stem Cells 26: 2257-2265.

14. Evseenko D, Schenke-Layland K, Dravid G, Zhu Y, Hao OL, et al. (2009) Identification of the critical extracellular matrix proteins that promote human embryonic stem cell assembly. Stem Cells Dev 18: 919-928.

15. Meng Y, Eshghi S, Li YJ, Schmidt R, Schaffer DV, et al. (2010) Characterization of integrin engagement during defined human embryonic stem cell culture. FASEB J 24: 1056-1065.

16. Nomizu M, Kim WH, Yamamura K, Utani A, Song SY, et al. (1995) Identification of cell binding sites in the laminin alpha 1 chain carboxyl-terminal globular domain by systematic screening of synthetic peptides. J Biol Chem 270: 20583-20590.

17. Ruoslahti E (1996) RGD and other recognition sequences for integrins. Annu Rev Cell Dev Biol 12: 697-715.

18. Pierschbacher MD, Ruoslahti E (1984) Gell attachment activity of fibronectin can be duplicated by small synthetic fragments of the molecule. Nature 309: $30-$ 33.

19. Ito Y, Kajihara M, Imanishi Y (1991) Materials for enhancing cell adhesion by immobilization of cell-adhesive peptide. J Biomed Mater Res 25: 1325-1337.

20. Burgeson RE, Chiquet M, Deutzmann R, Ekblom P, Engel J, et al. (1994) A new nomenclature for the laminins. Matrix Biol 14: 209-211.

21. Jin S, Yao H, Melkoumian Z, Ye K (2011) Expansion of human iPS cells on xeno-free, synthetic surface in defined medium. Proceeding of ISSCR annual meeting: 175 .

22. Derda R, Li L, Orner BP, Lewis RL, Thomson JA, et al. (2007) Defined substrates for human embryonic stem cell growth identified from surface arrays. ACS Chem Biol 2: 347-355.

23. Gerecht S, Burdick JA, Ferreira LS, Townsend SA, Langer R, et al. (2007) Hyaluronic acid hydrogel for controlled self-renewal and differentiation of human embryonic stem cells. Proc Natl Acad Sci U S A 104: 11298-11303.

\section{Author Contributions}

Conceived and designed the experiments: SJ ZM. Performed the experiments: HY SJ JW. Analyzed the data: SJ HY JW. Contributed reagents/materials/analysis tools: SJ ZM KY. Wrote the paper: SJ KY ZM.

24. Mahlstedt MM, Anderson D, Sharp JS, McGilvray R, Munoz MD, et al. (2010) Maintenance of pluripotency in human embryonic stem cells cultured on a synthetic substrate in conditioned medium. Biotechnol Bioeng 105: 130-140.

25. Klim JR, Li L, Wrighton PJ, Piekarczyk MS, Kiessling LL (2010) A defined glycosaminoglycan-binding substratum for human pluripotent stem cells. Nat Methods 7: 989-994.

26. Derda R, Musah S, Orner BP, Klim JR, Li L, et al. (2010) High-throughput discovery of synthetic surfaces that support proliferation of pluripotent cells. J Am Chem Soc 132: 1289-1295.

27. Nakajima M, Hayashi K, Katayama K, Amano Y, Egi Y, et al. (2003) Wf-536 prevents tumor metastasis by inhibiting both tumor motility and angiogenic actions. Eur J Pharmacol 459: 113-120.

28. Murata T, Arii S, Nakamura T, Mori A, Kaido T, et al. (2001) Inhibitory effect of Y-27632, a ROCK inhibitor, on progression of rat liver fibrosis in association with inactivation of hepatic stellate cells. J Hepatol 35: 474-481.

29. Weber JL, Dolley-Sonneville P, Weber DM, Fadeev AG, Zhou Y, et al. (2010) Corning $^{\circledR}$ Synthemax ${ }^{\mathrm{TM}}$ Surface: a tool for feeder-free, xeno-free culture of human embryonic stem cells. Nature Methods Dec.: an6-an7.

30. Pfannkuche K, Hannes T, Khalil M, Noghabi MS, Morshedi A, et al. (2010) Induced pluripotent stem cells: a new approach for physiological research. Cell Physiol Biochem 26: 105-124.

31. Chin MH, Mason MJ, Xie W, Volinia S, Singer M, et al. (2009) Induced pluripotent stem cells and embryonic stem cells are distinguished by gene expression signatures. Cell Stem Cell 5: 111-123.

32. Jin S, Yao H, Krisanarungson P, Haukas A, Ye K (2012) Porous Membrane Substrates Offer Better Niches to Enhance the Wnt Signaling and Promote Human Embryonic Stem Cell Growth and Differentiation. Tissue Eng Part A.

33. Jin S, Chen C, Montelaro RC (2005) Equine infectious anemia virus Gag p9 function in early steps of virus infection and provirus production. J Virol 79 : 8793-8801.

34. Jin S, Issel CJ, Montelaro RC (2004) Serological method using recombinant S2 protein to differentiate equine infectious anemia virus (EIAV)-infected and EIAV-vaccinated horses. Clin Diagn Lab Immunol 11: 1120-1129.

35. Jin S, Ellis E, Veetil JV, Yao H, Ye K (2011) Visualization of human immunodeficiency virus protease inhibition using a novel Forster resonance energy transfer molecular probe. Biotechnol Prog.

36. D'Amour KA, Bang AG, Eliazer S, Kelly OG, Agulnick AD, et al. (2006) Production of pancreatic hormone-expressing endocrine cells from human embryonic stem cells. Nat Biotechnol 24: 1392-1401.

37. Kroon E, Martinson LA, Kadoya K, Bang AG, Kelly OG, et al. (2008) Pancreatic endoderm derived from human embryonic stem cells generates glucose-responsive insulin-secreting cells in vivo. Nat Biotechnol 26: 443-452.

38. Van Hoof D, DAmour KA, German MS (2009) Derivation of insulin-producing cells from human embryonic stem cells. Stem Cell Res 3: 73-87.

39. Rowland TJ, Miller LM, Blaschke AJ, Doss EL, Bonham AJ, et al. (2010) Roles of integrins in human induced pluripotent stem cell growth on Matrigel and vitronectin. Stem cells and development 19: 1231-1240.

40. Grashoff C, Hoffman BD, Brenner MD, Zhou R, Parsons M, et al. (2010) Measuring mechanical tension across vinculin reveals regulation of focal adhesion dynamics. Nature 466: 263-266.

41. Galbraith CG, Yamada KM, Sheetz MP (2002) The relationship between force and focal complex development. J Cell Biol 159: 695-705.

42. Riveline D, Zamir E, Balaban NQ, Schwarz US, Ishizaki T, et al. (2001) Focal contacts as mechanosensors: externally applied local mechanical force induces growth of focal contacts by an mDial-dependent and ROCK-independent mechanism. J Cell Biol 153: 1175-1186.

43. van der Gaag EJ, Leccia MT, Dekker SK, Jalbert NL, Amodeo DM, et al. (2002) Role of zyxin in differential cell spreading and proliferation of melanoma cells and melanocytes. The Journal of investigative dermatology 118: 246-254.

44. Peerani R, Rao BM, Bauwens C, Yin T, Wood GA, et al. (2007) Nichemediated control of human embryonic stem cell self-renewal and differentiation. The EMBO journal 26: 4744-4755.

45. Shi Y, Massague J (2003) Mechanisms of TGF-beta signaling from cell membrane to the nucleus. Cell 113: 685-700.

46. Rao BM, Zandstra PW (2005) Culture development for human embryonic stem cell propagation: molecular aspects and challenges. Current opinion in biotechnology 16: 568-576. 
47. Besser D (2004) Expression of nodal, lefty-a, and lefty-B in undifferentiated human embryonic stem cells requires activation of Smad2/3. The Journal of biological chemistry 279: 45076-45084.

48. James D, Levine AJ, Besser D, Hemmati-Brivanlou A (2005) TGFbeta/activin/ nodal signaling is necessary for the maintenance of pluripotency in human embryonic stem cells. Development 132: 1273-1282.

49. Xu RH, Peck RM, Li DS, Feng X, Ludwig T, et al. (2005) Basic FGF and suppression of BMP signaling sustain undifferentiated proliferation of human ES cells. Nature Methods 2: 185-190.

50. Bendall SC, Stewart MH, Bhatia M (2008) Human embryonic stem cells: lessons from stem cell niches in vivo. Regenerative medicine 3: 365-376.

51. Liu M, Tanswell AK, Post M (1999) Mechanical force-induced signal transduction in lung cells. Am J Physiol 277: L667-683.

52. Bresnick AR (1999) Molecular mechanisms of nonmuscle myosin-II regulation. Curr Opin Cell Biol 11: 26-33.

53. Solomon F, Magendantz M (1981) Cytochalasin separates microtubule disassembly from loss of asymmetric morphology. J Cell Biol 89: 157-161.

54. Somlyo AP, Somlyo AV (2000) Signal transduction by G-proteins, rho-kinase and protein phosphatase to smooth muscle and non-muscle myosin II. J Physiol 522 Pt 2: 177-185.

55. Bershadsky AD, Balaban NQ, Geiger B (2003) Adhesion-dependent cell mechanosensitivity. Annu Rev Cell Dev Biol 19: 677-695.

56. Vuoristo S, Virtanen I, Takkunen M, Palgi J, Kikkawa Y, et al. (2009) Laminin isoforms in human embryonic stem cells: synthesis, receptor usage and growth support. Journal of cellular and molecular medicine 13: 2622-2633.

57. Katsumi A, Orr AW, Tzima E, Schwartz MA (2004) Integrins in mechanotransduction. J Biol Chem 279: 12001-12004.

58. Stupack DG (2007) The biology of integrins. Oncology (Williston Park) 21: 6-12.
59. Yeung T, Georges PC, Flanagan LA, Marg B, Ortiz M, et al. (2005) Effects of substrate stiffness on cell morphology, cytoskeletal structure, and adhesion. Cell Motil Cytoskeleton 60: 24-34.

60. Dalby MJ, Riehle MO, Johnstone H, Affrossman S, Curtis AS (2002) In vitro reaction of endothelial cells to polymer demixed nanotopography. Biomaterials 23: 2945-2954.

61. Markert LD, Lovmand J, Foss M, Lauridsen RH, Lovmand M, et al. (2009) Identification of distinct topographical surface microstructures favoring either undifferentiated expansion or differentiation of murine embryonic stem cells. Stem Cells Dev 18: 1331-1342.

62. Kantawong F, Burgess KE, Jayawardena K, Hart A, Riehle MO, et al. (2010) Effects of a surface topography composite with puerariae radix on human STRO-1-positive stem cells. Acta Biomater 6: 3694-3703.

63. Berry CC, Campbell G, Spadiccino A, Robertson M, Curtis AS (2004) The influence of microscale topography on fibroblast attachment and motility. Biomaterials 25: 5781-5788.

64. McNamara LE, McMurray RJ, Biggs MJP, Kantawong F, Oreffo ROC, et al. (2010) Nanotopographical control of stem cell differentiation. J Tissue Eng 2010: $1-13$.

65. Geiger B, Bershadsky A (2001) Assembly and mechanosensory function of focal contacts. Curr Opin Cell Biol 13: 584-592.

66. Balaban NQ, Schwarz US, Riveline D, Goichberg P, Tzur G, et al. (2001) Force and focal adhesion assembly: a close relationship studied using elastic micropatterned substrates. Nat Cell Biol 3: 466-472.

67. Geiger B, Bershadsky A, Pankov R, Yamada KM (2001) Transmembrane crosstalk between the extracellular matrix-cytoskeleton crosstalk. Nat Rev Mol Cell Biol 2: 793-805. 